Research Article

\title{
Numerical Study on Rod Thrust Vector Control for Physical Applications
}

\author{
Dong $\mathrm{Li}^{1}$ and Kexin $\mathrm{Wu} \mathbb{D}^{2}$ \\ ${ }^{1}$ Huanghuai University, China \\ ${ }^{2}$ Zhejiang Sci-Tech University, China \\ Correspondence should be addressed to Kexin Wu; kexin.wu@zstu.edu.cn
}

Received 23 July 2021; Accepted 20 October 2021; Published 17 November 2021

Academic Editor: Kan Xie

Copyright (c) 2021 Dong Li and Kexin Wu. This is an open access article distributed under the Creative Commons Attribution License, which permits unrestricted use, distribution, and reproduction in any medium, provided the original work is properly cited.

\begin{abstract}
Mechanical thrust vector control is a classical and significant branch in the thrust vector control field, offering an extremely reliable control effect. In this article, steady-state and unsteady-state aerodynamic characteristics of the rod thrust vector control technology are numerically investigated in a two-dimensional supersonic nozzle. Complex flow phenomena caused by the penetrating rod in the diverging part of the supersonic nozzle are elucidated with the purpose of a profound understanding of this simple flow control technique for physical applications. Published experimental data are used to validate the dependability of current computational fluid dynamics results. A grid sensitivity study is carried through and analyzed. The result section discusses the impacts of two important factors on steady-state aerodynamic features, involving the rod penetration height and the rod location. Furthermore, unsteady-state flow features are analyzed under various rod penetration heights for the first time. Significant vectoring performance variations and flow topology descriptions are illuminated in full detail. While the rod penetration height increases, the vectoring angle increases, whereas the thrust coefficient decreases. As the rod location moves downstream close to the nozzle exit, the vectoring angle and thrust coefficient increase. In terms of unsteady-state aerodynamic effects, certain pressure oscillations occur upstream of the rod, which resulted from the expanding and shrinking of the upstream anticlockwise separation bubbles.
\end{abstract}

\section{Introduction}

One of the foremost issues for supersonic aircraft is attitude control. Advanced thrust vector controls are aimed at steering aircraft flexibly by generating side forces [1]. Thrust vector control technologies commonly have two categories, namely, fluidic and mechanical vectoring controls [2]. Figures $1(\mathrm{a})-1(\mathrm{~g})$ show seven representative fluidic control techniques employing a gas or liquid injection, involving coflow [3,4], counterflow [5-8], shock vector [9-12], bypass shock vector [13, 14], throat shifting [15-17], dual throat nozzle $[18,19]$, and bypass dual throat nozzle $[20,21]$. The above fluidic techniques have some outstanding advantages, for example, fast response, simple mechanical structure, and less thrust loss $[10,11]$. However, some big storage tanks used to store and offer gas or liquid injectant and other assorted equipment increase the weight of the entire system remarkably. Moreover, once the gas or liquid injectant is not sufficient, the control effectiveness would become unstable. As a consequence, these techniques are less attractive for various long-distance or long-time flights. Figures 1(h)$1(\mathrm{k})$ exhibit five traditional mechanical vectoring control technologies, including jet vane [22], movable nozzle [23], axial movable plate [24], and jet table [25]. The benefits of these mechanical vectoring control methods are offering an extremely dependable control effect. Nevertheless, overweight components and overcomplicated structures limit further development in future applications. To overcome the above techniques' limitations, a new idea using a simple rod is explored to control the vectoring angle, as shown in Figure 1(1) [26].

The CFD evaluation of this advanced method was carried through by Tiarn and Cavalleri [27] under an assumption of infinite probe penetration height. They expounded that this potential technique has adequate 


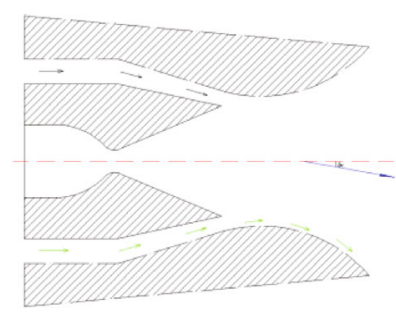

(a) Coflow

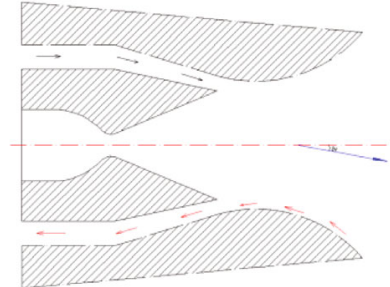

(b) Counterflow

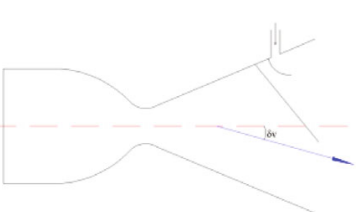

(c) Shock vector

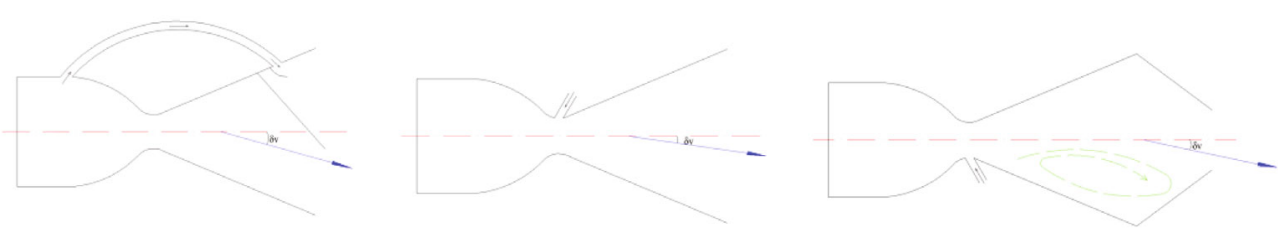

(d) Bypass shock vector

(e) Throat shifting

(f) Dual throat nozzle

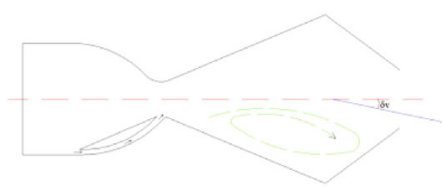

(g) Bypass dual throat nozzle

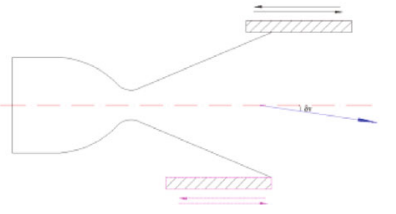

(j) Axial movable plate

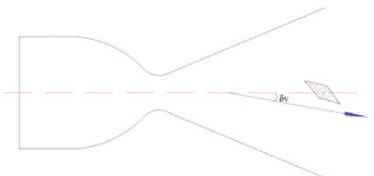

(h) Jet vane

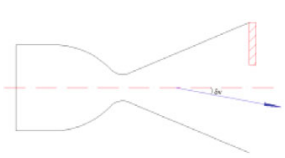

(k) Jet tab

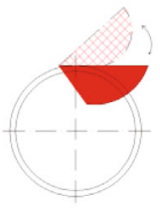

(i) Movable nozzle

Figure 1: Fluidic and mechanical thrust vector control principles.

capacity to generate a required thrust and saves much weight and cost. Cavalleri et al. [28] experimentally and theoretically investigated multiple probe control technology mounted on a flat plate in a supersonic freestream and found that a quick response is gained. Cavalleri [29] testified excellent pitching and yawing control effectiveness using four probes with an interval of $90^{\circ}$. Mokhtari et al. [30] experimentally studied the impact of a single-rod penetration height on the system performance for an axisymmetric nozzle and found that it affects the vectoring angle remarkably. Subsequently, Babaeyan and Hojaji [31] experimentally investigated a new dual-rod penetration technique in the opposite direction to control the flow deflection and illustrated that dual-rod penetration can obtain a maximum vectoring angle of 4.35 degrees, whereas the axial thrust declines up to $5.5 \%$ owing to the occurrence of various opposite shocks. Srinivas and Sridhar [32] experimentally explored the feasibility of a single rectangular strut on the vectoring performance of the axisymmetric nozzle and elucidated that it can achieve an effective control by changing the strut penetration height.

Most of the previous researches focused on the experimental test. The CFD means are used herein to illuminate the flow-field structures in full detail. Specifically, a series of CFD work has been conducted to illustrate the impacts of rod penetration height and rod location on the steady- state performance in a two-dimensional (2-D) supersonic nozzle. Additionally, the flow oscillations caused by the expanding and shrinking of the separation bubbles upstream of the rod are expounded to testify to the effectiveness of the rod thrust vector control. The abovementioned aerodynamic characteristics are numerically investigated, and quite meaningful results are obtained.

\section{Fundamentals and Numerical Analysis}

2.1. Basic Flow Characteristics. The flow separations in a supersonic freestream are always accompanied by various shocks that cause significant adverse pressure gradients on the boundary layer. The shock-wave/boundary-layer interaction principle plays an important role in these complex flow separations, which is strongly linked to low-frequency oscillations of separation shocks [33,34]. Figure 2 depicts the schematic of flow phenomena in a supersonic nozzle with a rod inserted through the nozzle top wall. A separation shock forms upstream of the rod [35-37]. Due to this separation shock, a sudden jump in pressure leads to an adverse pressure gradient in the boundary layer. Then, the boundary layer separates and the flow inside goes up away from the wall. This whole process is called the separation of the boundary layer from the wall. Straight ahead of the rod, a 


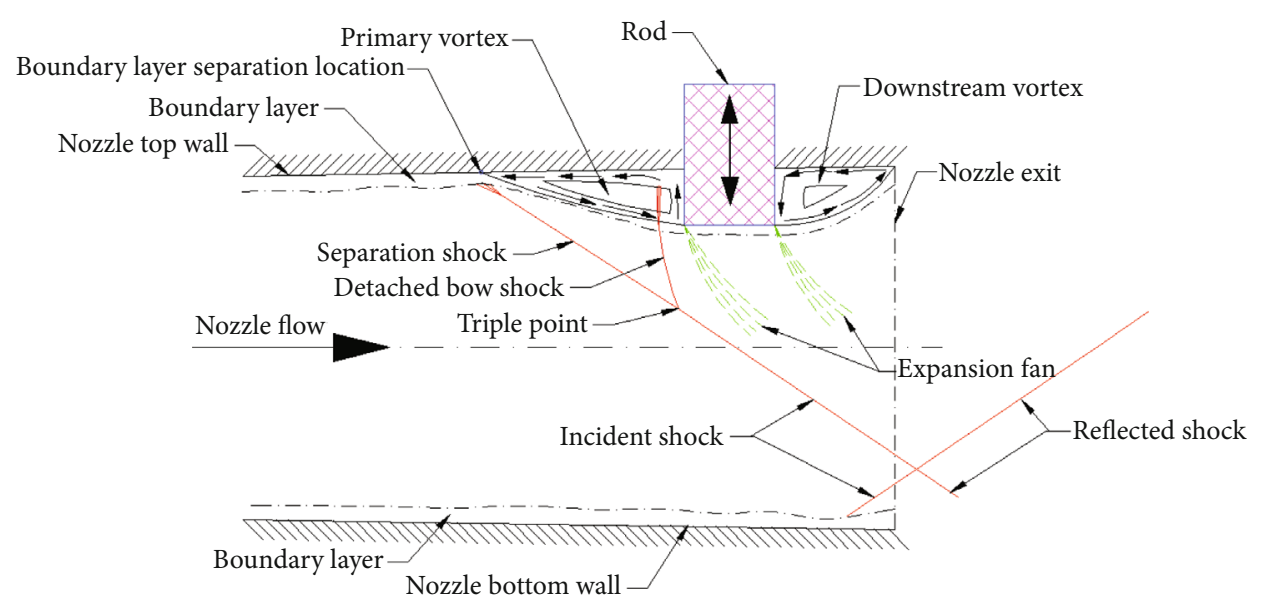

Figure 2: Schematic of flow features around the rod in a supersonic nozzle.

detached bow shock stands a small distance away from the rod. An anticlockwise rotating vortex which is named primary vortex forms inside the boundary layer. The separation and detached bow shocks crash each other at the triple point and merge into an incident shock. This incident shock impinges on the bottom nozzle wall, resulting in another boundary layer separation and the new reflected shocks. Expansion fans form at the forward and rearward edges of the rod, respectively, which change the flow direction toward the top wall side and accelerate the flow velocity. At the back of the rod, anticlockwise rotating downstream vortex forms inside the boundary layer. All the above flow phenomena are strongly relying on the rod penetration height, rod location, and upstream stagnation pressure. Since the variations of the static pressure are associated with the abovementioned shocks, imbalanced pressure distributions are obtained along the top and bottom nozzle walls. Then, the net side force is generated to deflect the flow direction.

Critical parameters are utilized to quantify system performance. The vectoring angle, $\delta_{\mathrm{v}}$, is determined by horizontal force, $F_{x}$, and vertical force, $F_{y}$, as shown in the following equation:

$$
\delta_{v}=\tan ^{-1}\left(F_{\mathrm{y}} / F_{\mathrm{x}}\right)
$$

The net thrust force is made up of momentum flux and surface force, as illustrated in Equations (2) and (3).

$$
\begin{aligned}
& F_{y}=\dot{m} \bullet U_{\mathrm{e} y}, \\
& F_{x}=\dot{m} \cdot U_{\mathrm{ex}}+\left(P_{\mathrm{e}}-P_{\mathrm{atm}}\right) \bullet A_{\mathrm{e}},
\end{aligned}
$$

where $\dot{m}$ represents the mass flow rate, $U_{\text {ex }}$ denotes the horizontal velocity, and $U_{\mathrm{e} y}$ is the vertical velocity. $P_{\mathrm{e}}$ represents the area-weighted average pressure on the nozzle exit, and $A_{\mathrm{e}}$ means the exit area. $P_{\text {atm }}$ represents the atmosphere pressure $(101325 \mathrm{~Pa})$.

Another coefficient is defined to assess the thrust loss, namely, thrust coefficient, $C_{\mathrm{t}}$. It can be expressed as the ratio of real thrust, $F_{\mathrm{r}}$, and theoretically isentropic thrust, $F_{\mathrm{i}}$, as shown in Equations (4) and (5).

$$
\begin{aligned}
C_{\mathrm{t}}=\frac{F_{\mathrm{r}}}{F_{\mathrm{i}}} & =\frac{\sqrt{F_{x}^{2}+F_{y}^{2}}}{F_{\mathrm{i}}}, \\
F_{\mathrm{i}, p} & =\dot{m} \sqrt{\frac{2 \gamma R_{\mathrm{g}} T_{0}}{\gamma-1}\left[1-\left(\frac{P_{\mathrm{atm}}}{P_{0}}\right)^{(\gamma-1) / \gamma}\right]} .
\end{aligned}
$$

2.2. Governing Equations. The classical Reynolds-averaged Navier-Stokes (RANS) approach is used in this article. Critical equations in terms of mass, momentum, and energy are given as follows:

Mass conservation

$$
\frac{\partial \rho}{\partial t}+\frac{\partial}{\partial x_{i}}\left(\rho u_{i}\right)=0
$$

Momentum conservation

$$
\frac{D u}{D t}\left(\rho u_{i}\right)-\frac{\partial p}{\partial x_{i}}+\frac{\partial}{\partial x_{j}}\left[\mu_{e f f}\left(\frac{\partial u_{i}}{\partial x_{j}}+\frac{\partial u_{j}}{\partial x_{i}}-\frac{2}{3} \delta_{i j} \frac{\partial u_{k}}{\partial x_{k}}\right)\right]+\frac{\partial}{\partial x_{j}}\left(-\rho \overline{u_{i}^{\prime} u_{j}^{\prime}}\right)=0
$$

Energy conservation

$$
(\rho E)+\frac{\partial}{\partial x_{i}}\left[u_{i}(\rho E+p)\right]=\frac{\partial}{\partial x_{i}}\left[\left(\alpha+\frac{C_{p} u_{t}}{P_{r t}}\right) \frac{\partial T}{\partial x_{i}}+u_{j}\left(\tau_{i j}\right)_{\mathrm{eff}}\right],
$$

where $\tau_{i j}$ represents the stress tensor, which can be expressed as in the following equation:

$$
\tau_{i j}=\mu_{\mathrm{eff}}\left(\frac{\partial u_{j}}{\partial x_{i}}+\frac{\partial u_{i}}{\partial x_{j}}\right)-\frac{2}{3} \mu_{\mathrm{eff}} \frac{\partial u_{i}}{\partial x_{i}} \delta_{i j}
$$




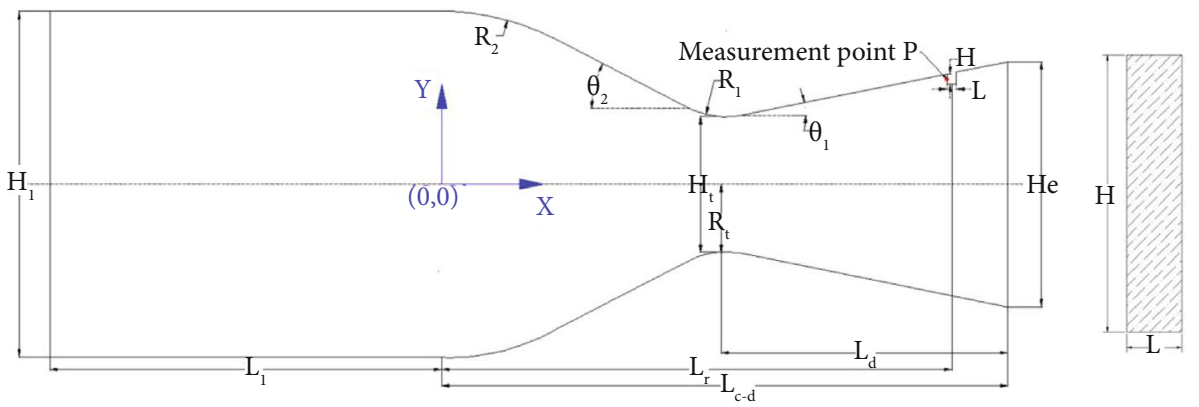

Figure 3: Geometry description of the CFD model.

TABle 1: Detailed geometry description.

\begin{tabular}{lr}
\hline Geometry parameters & Value \\
\hline Inlet height, $H_{1}$ & $70.4 \mathrm{~mm}$ \\
Nozzle exit height, $H_{\mathrm{e}}$ & $49.38 \mathrm{~mm}$ \\
Nozzle throat height, $H_{\mathrm{t}}$ & $27.48 \mathrm{~mm}$ \\
Rod length, $L$ & $2 \mathrm{~mm}$ \\
Converging-diverging section length, $L_{\mathrm{c}-\mathrm{d}}$ & $115.57 \mathrm{~mm}$ \\
Diverging section length, $L_{\mathrm{d}}$ & $57.97 \mathrm{~mm}$ \\
Distance between the rod center and the original point, $L_{\mathrm{r}}$ & $104.14 \mathrm{~mm}$ \\
Throat transition arc radius, $R_{1}$ & $15.89 \mathrm{~mm}$ \\
Converging part transition arc radius, $R_{2}$ & $50.77 \mathrm{~mm}$ \\
Nozzle throat radius, $R_{\mathrm{t}}$ & $13.74 \mathrm{~mm}$ \\
Diverging angle, $\theta_{1}$ & $11.01^{\circ}$ \\
Converging angle, $\theta_{2}$ & $27.29^{\circ}$ \\
\hline
\end{tabular}

The perfect gas law which is shown in Equation (10) can be utilized to close the entire equation system.

$$
\frac{p}{\rho}=R_{\mathrm{g}} T
$$

2.3. Numerical Schemes and Boundary Conditions. The geometry dimensions of the 2-D supersonic nozzle with an inserted rod are shown schematically in Figure 3. This basic geometry model is referred to as Waithe and Deere's experiment [38], in which the detailed dimensions are listed in Table 1. The design Mach number of the nozzle is $M=2$. The left chamber filled with high-pressure gas has a height of $H_{1}=70.4 \mathrm{~mm}$ and a length of $L_{1}=80 \mathrm{~mm}$. The convergent-divergent portion of the supersonic nozzle has a total length of $L_{\mathrm{c}-\mathrm{d}}=115.57 \mathrm{~mm}$; thereinto, the diverging section length is $L_{\mathrm{d}}=57.97 \mathrm{~mm}$. The nozzle throat height is $H_{\mathrm{t}}=27.48 \mathrm{~mm}$; namely, the nozzle throat radius is $R_{\mathrm{t}}=$ $13.74 \mathrm{~mm}$, and the nozzle exit height is $H_{\mathrm{e}}=49.38 \mathrm{~mm}$. The diverging and converging angles are $\theta_{1}=11.01^{\circ}$ and $\theta_{2}$ $=27.29^{\circ}$, respectively. A solid rod is inserted into the diverging part of the nozzle, in which the horizontal distance between the original point and the axis of the $\operatorname{rod}$ is $L_{\mathrm{r}}=$ $104.14 \mathrm{~mm}$. A measurement point is located in the middle of the rod penetration height to record the static pressure history in unsteady simulations.
Figure 4 shows the computational domain, grid, and boundary conditions, simultaneously. Since the 2-D flow field is asymmetrical, a full domain is used. At the nozzle exit, an extension domain whose length and height are $50 R_{t}$ and $40 R_{t}$ is considered to ensure a fully developed jet flow. The multiblock technique is utilized to segment the whole domain, and purely structured grids are created for each block to predict the flow-field details accurately. Because of the impact of the gas viscosity, the boundary layer grid is created and distributed along with the internal nozzle wall, as shown in Figures 4(b)-4(d). The maximum wall $y+$ value is less than 1 . The mesh refinement is specifically used in some zones, involving the nozzle throat, the area around the rod, and the nozzle exit. After the nozzle exit, a gradually sparse grid distribution is used to reduce the total grid cells while ensuring full flow development. Suitable boundary conditions are considered, including pressure inlet, pressure outlet, and wall. The incoming flow inlet connected with the combustor is defined as the pressure inlet. The nozzle and rod surfaces are selected as no-slip and adiabatic walls. Other boundary conditions are set as the pressure outlet. All CFD work is carried through for a total temperature of $T_{0}=300 \mathrm{~K}$, and the value of NPR is 4.6.

ANSYS Fluent 2020 R2 is used to solve steady and unsteady RANS equations. The solver type is a densitybased solver, and the velocity formulation is selected as 


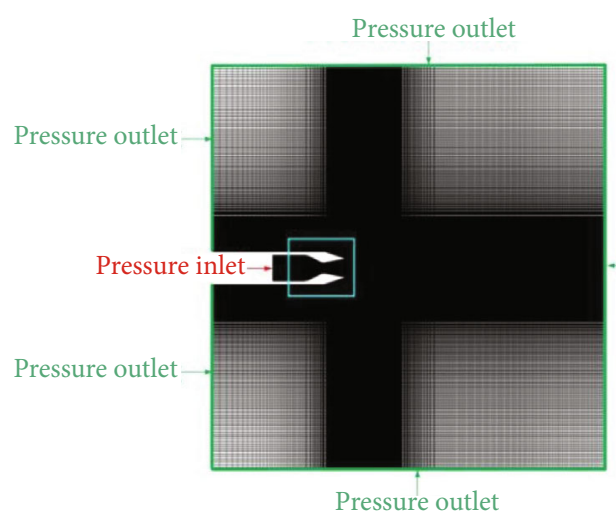

(a)

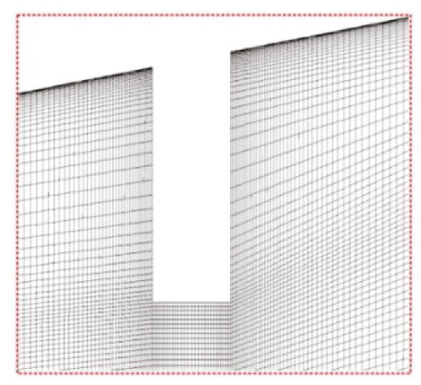

(c)

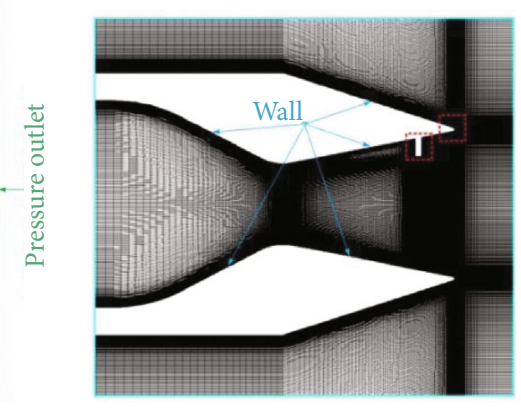

(b)

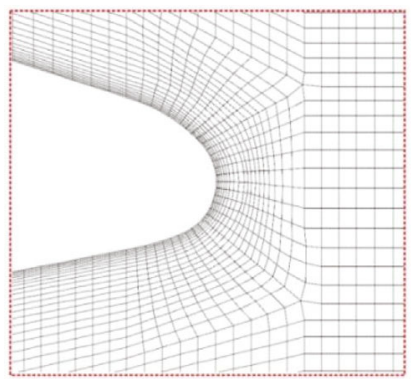

(d)

Figure 4: Computational domain and boundary conditions: (a) overall view; (b) local view; (c) zoom-in view around the rod; (d) zoom-in view close to the nozzle exit.

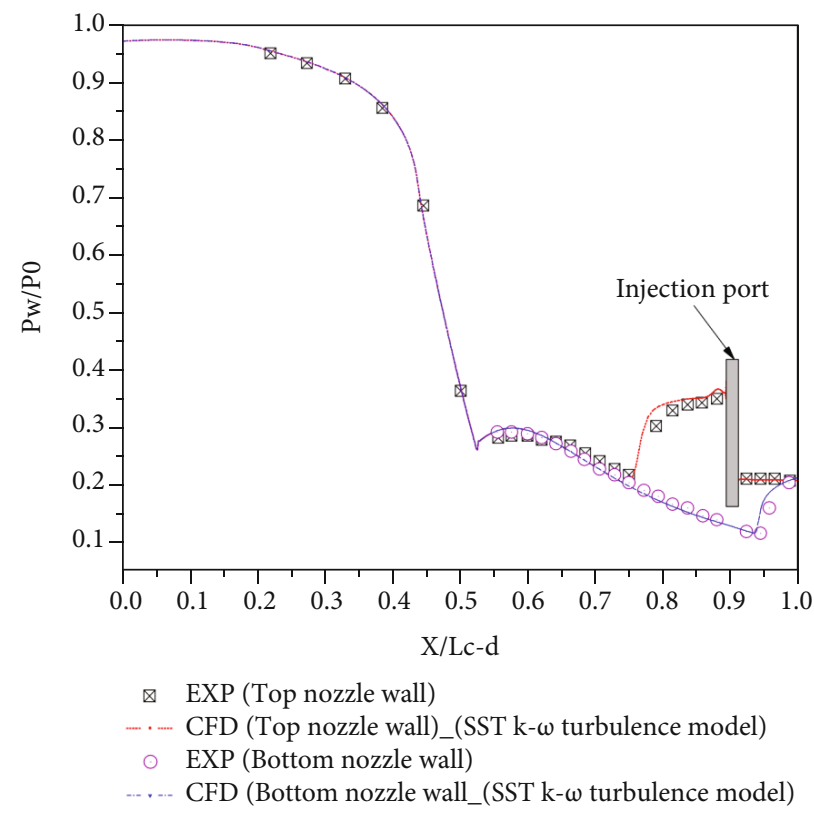

FIgURE 5: Experimental validations of static pressures along wall surfaces $(\mathrm{NPR}=4.6)$.

absolute. The working fluid is an ideal gas whose gas viscosity is calculated based on Sutherland's law, and the operating pressure is set at zero. For the spatial discretization, the leastsquares cell-based gradient combined with second-order upwind schemes in terms of flow, turbulent kinetic energy, and specific dissipation rate is chosen. Implicit formulation and a flux type of AUSM are adopted to increase the courant number rapidly and effectively. Since the courant number and underrelaxation factors are sensitive and important in the calculation process, a gradually increasing trend is 


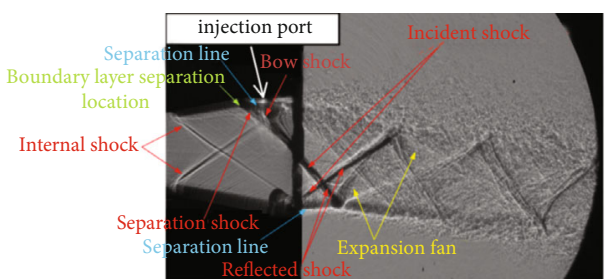

(a)

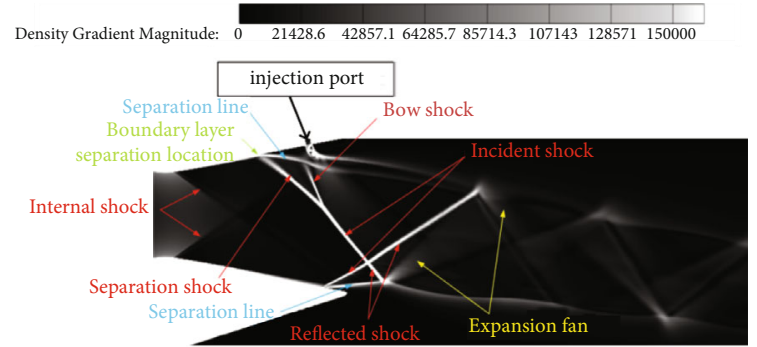

(b)

FIGURE 6: Comparison between experimental shadowgraph and CFD results: (a) experimental shadowgraph; (b) CFD.

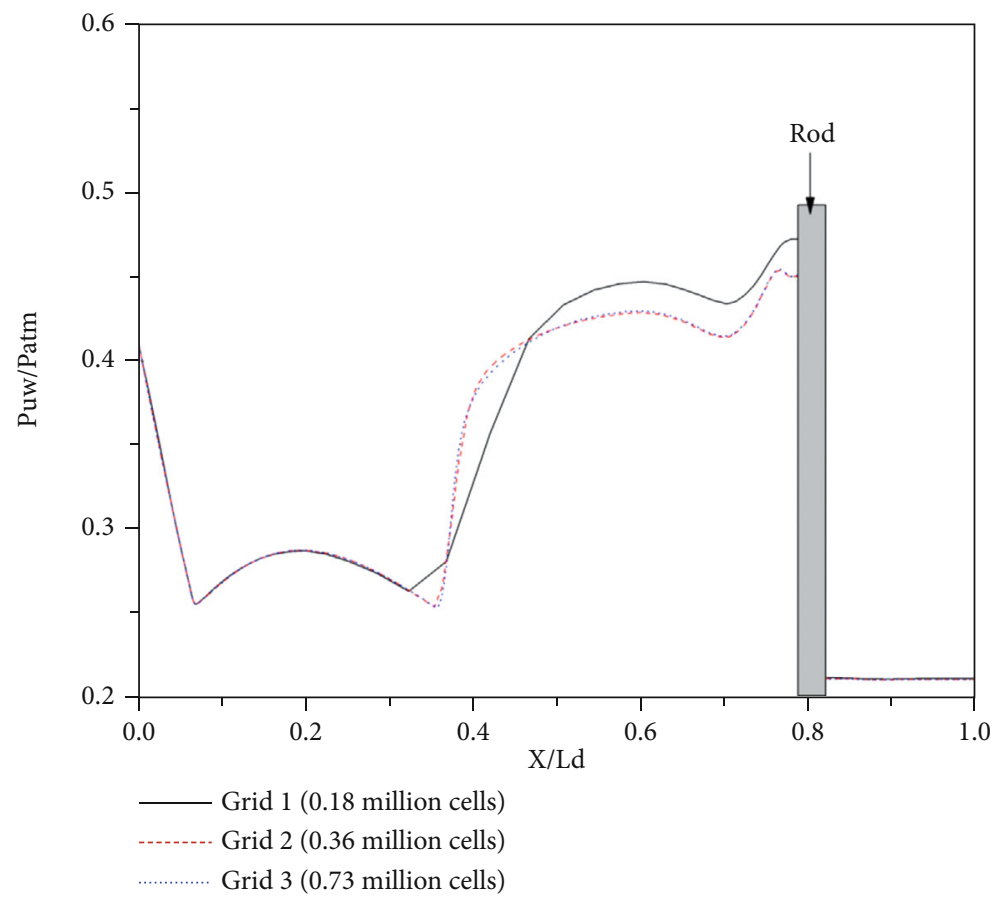

FIGURE 7: Static pressure distributions along the diverging part on the top wall for grid independence study $\left(\mathrm{NPR}=4.6, H / R_{\mathrm{t}}=0.58\right.$, and $\left.X_{\mathrm{r}} / L_{\mathrm{d}}=0.8\right)$.

adopted for steady simulations. After obtaining the steady CFD results, the unsteady simulation is continued to run with an adaptive time step size of $1 e-06$.

Concerning the turbulence model, three main categories are Spalart-Allmaras (S-A), $k-\varepsilon$, and $k-\omega$, respectively. The S-A model has prominent benefits about convergence and stability; however, it could not precisely forecast characteristics of shear flow, separation flow, and turbulence flow. The $k-\varepsilon$ model has the outstanding advantages of predicting the external flow; nevertheless, it could not accurately project the flow field having the adverse pressure gradient and jet flow. The $k-\omega$ model is extremely effective in predicting the separation flow, internal flow, and jet flow. In particular, the SST $k-\omega$ model remarkably strengthens the prediction accuracy of the $k-\omega$ model for some cases away from the wall. In consideration of present issues covering internal flow, separation flow, adverse pressure gradient, and jet flow, the SST $k-\omega$ model is quite appropriate. Moreover, some previous CFD researches have proved the abovementioned advantages of the SST $k-\omega$ model [9-12, 19, 39]. Consequently, the SST $k-\omega$ turbulence model is chosen here.

2.4. Validation. Experimental verification is essential to verify the validity of the turbulence model and numerical means. Since the rod thrust vector control technique is proposed from the shock vector control and there is no experiment concerning the planar rod thrust vector control, the present validation is referred to the experimental results from the shock vector control experiment. All present experimental data are cited from Waithe and Deere's article [38], in which normalized static pressure values along with the top and bottom walls of the converging-diverging (C-D) nozzle are offered. The experiment is carried through in the context of $\mathrm{NPR}=4.6$. Figure 5 displays comparative results with CFD and experimental means. The quantitatively contrast result shows that a very good consistency of 


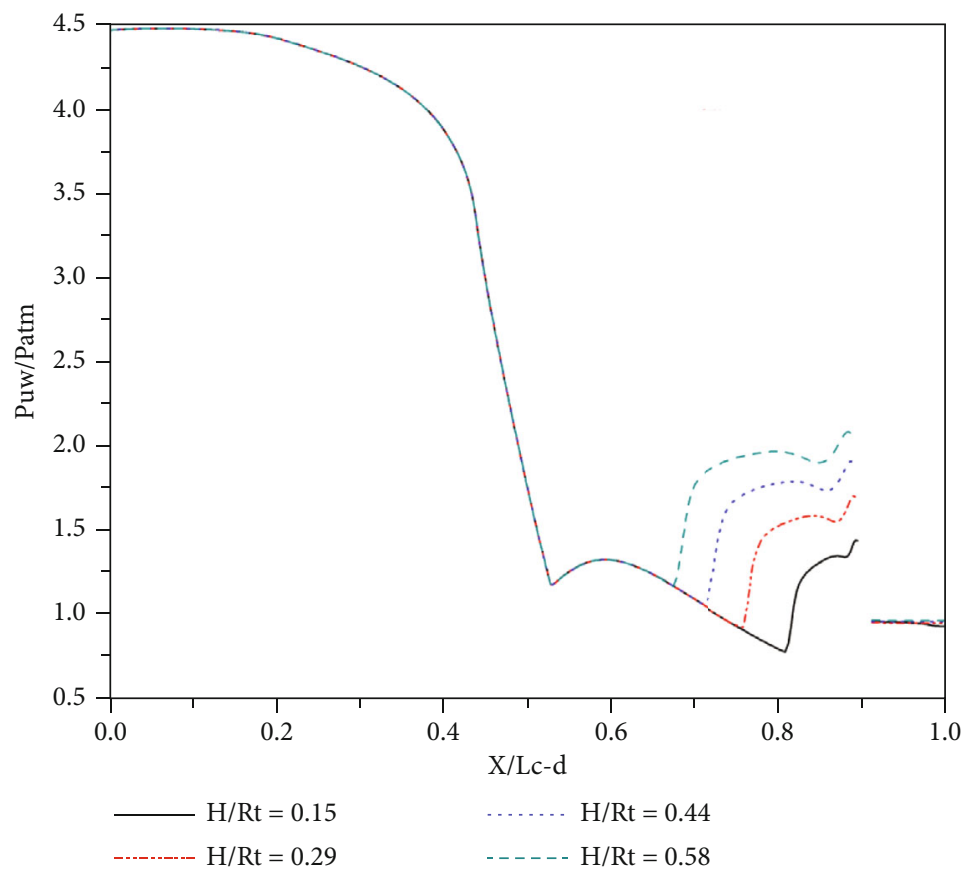

FIGURE 8: Static pressure distributions along the top wall for diverse rod penetration heights $\left(\mathrm{NPR}=4.6\right.$ and $\left.X_{\mathrm{r}} / L_{\mathrm{c}-\mathrm{d}}=0.8\right)$.

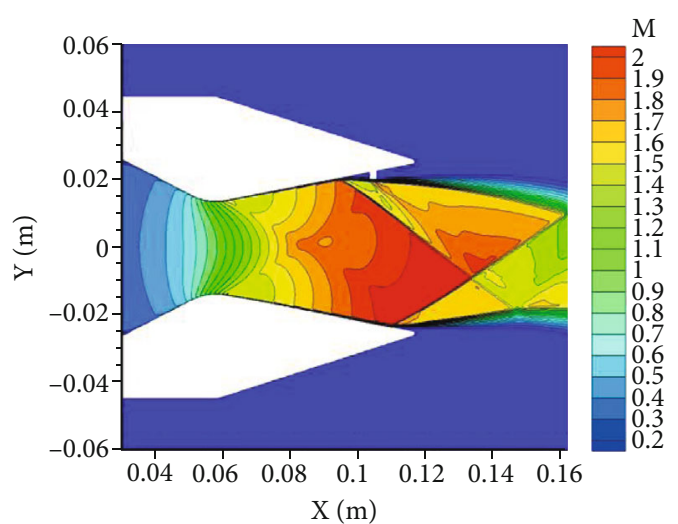

(a)

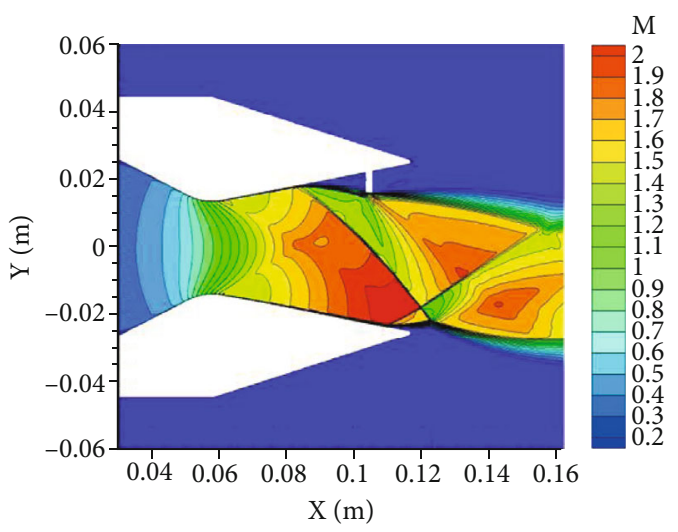

(c)

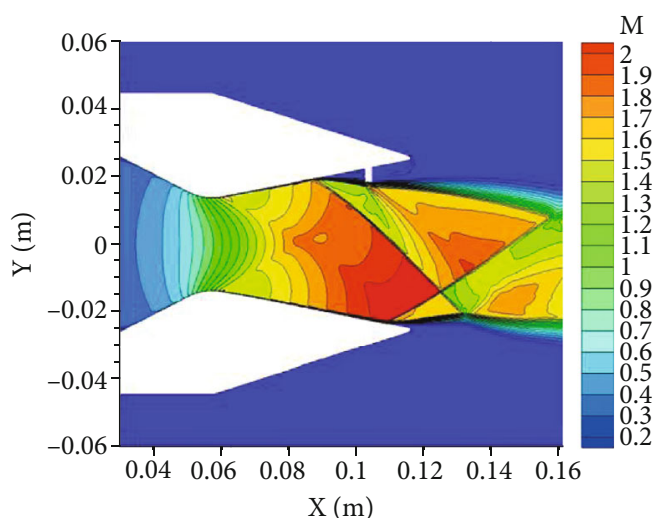

(b)

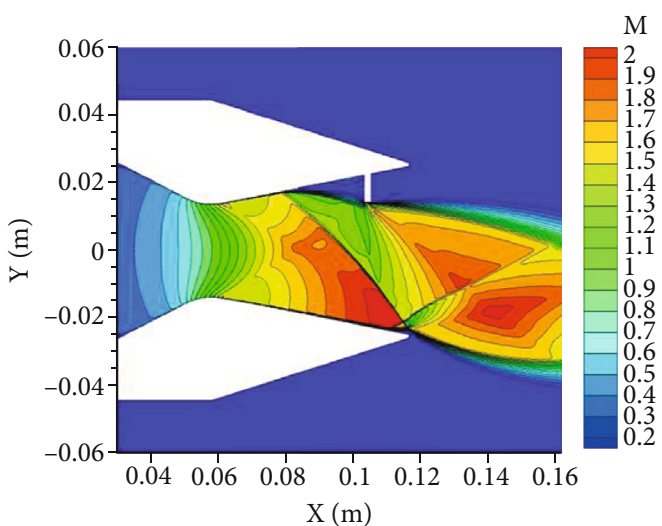

(d)

Figure 9: Mach contours for diverse rod penetration heights $\left(\mathrm{NPR}=4.6\right.$ and $\left.X_{\mathrm{r}} / L_{\mathrm{d}}=0.8\right)$ : (a) $H / R_{\mathrm{t}}=0.15$; (b) $H / R_{\mathrm{t}}=0.29$; (c) $H / R_{\mathrm{t}}=0.44$; (d) $H / R_{\mathrm{t}}=0.58$. 


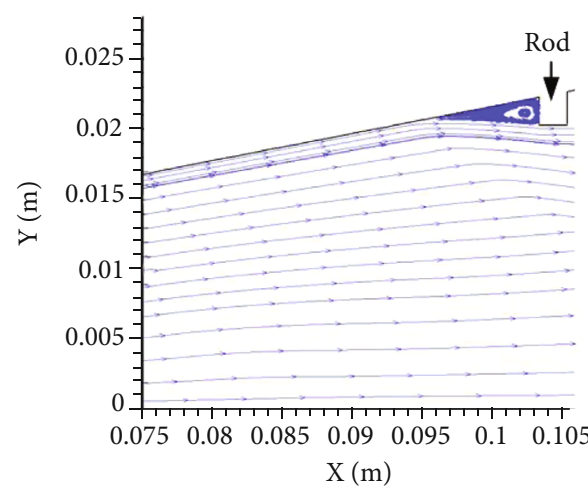

(a)

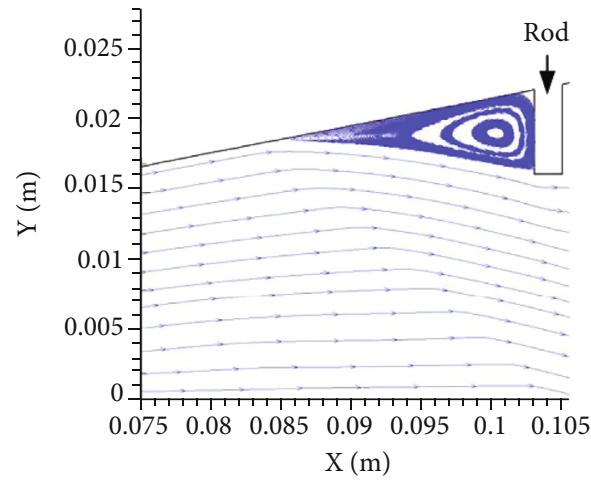

(c)

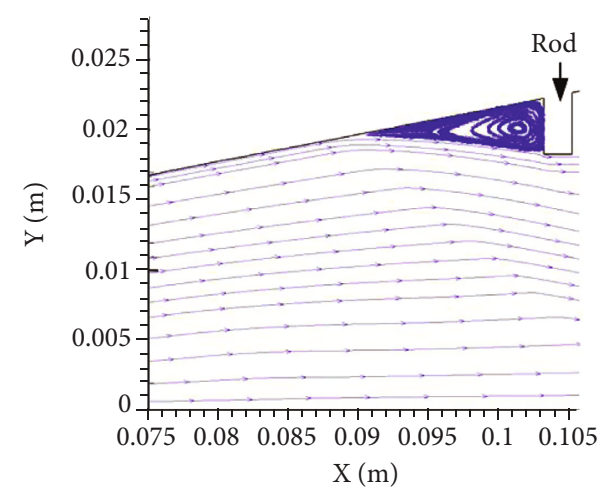

(b)

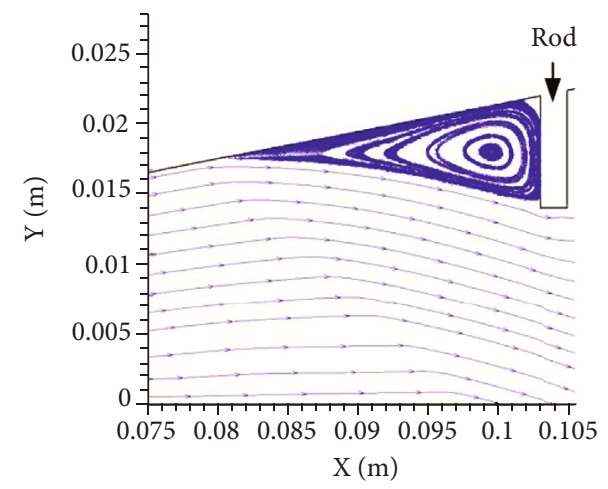

(d)

FiguRE 10: Streaklines for diverse rod penetration heights (NPR $=4.6$ and $\left.X_{\mathrm{r}} / L_{\mathrm{d}}=0.8\right)$ : (a) $H / R_{\mathrm{t}}=0.15$; (b) $H / R_{\mathrm{t}}=0.29$; (c) $H / R_{\mathrm{t}}=0.44$; (d) $H / R_{\mathrm{t}}=0.58$.

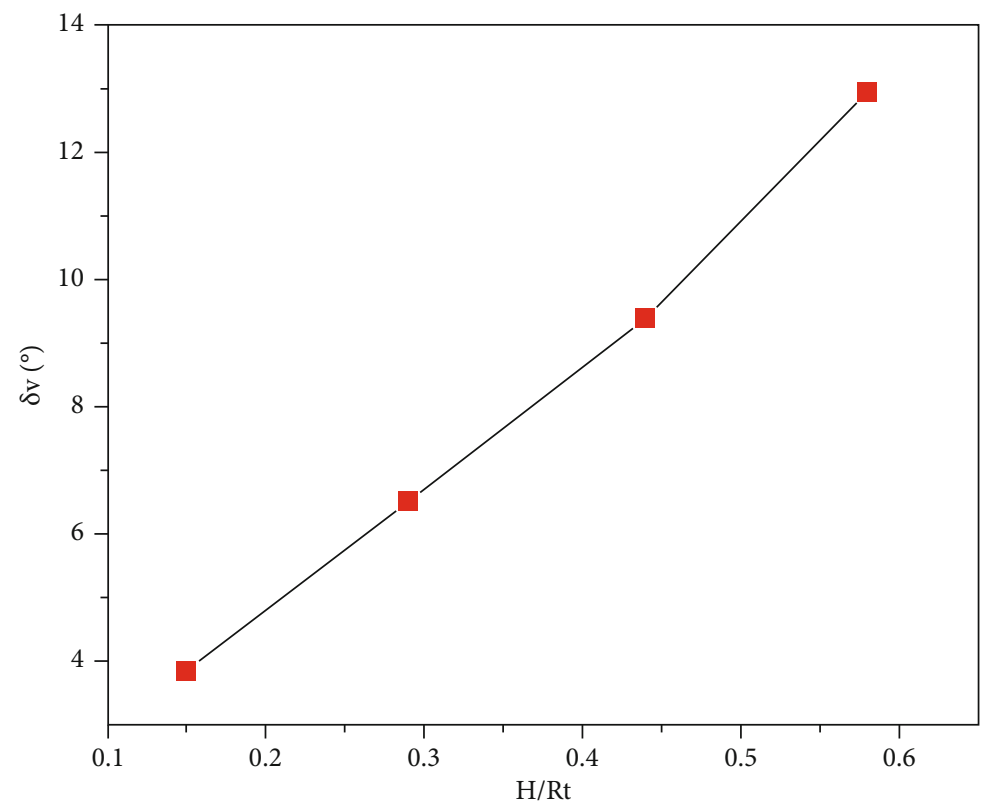

FiguRE 11: Vectoring angles for diverse rod penetration heights $\left(\mathrm{NPR}=4.6\right.$ and $\left.X_{\mathrm{r}} / L_{\mathrm{d}}=0.8\right)$.

normalized static pressures on top and bottom walls is obtained. Synchronously, a qualitative comparison of the experimental shadowgraph captured by a high-speed camera with the density gradient contour is illustrated in Figure 6.
Corresponding physical phenomena are marked in both figures, including internal shocks, separation shocks, bow shocks, incident shocks, reflected shocks, boundary layer separation locations, separation lines, and expansion fans. 


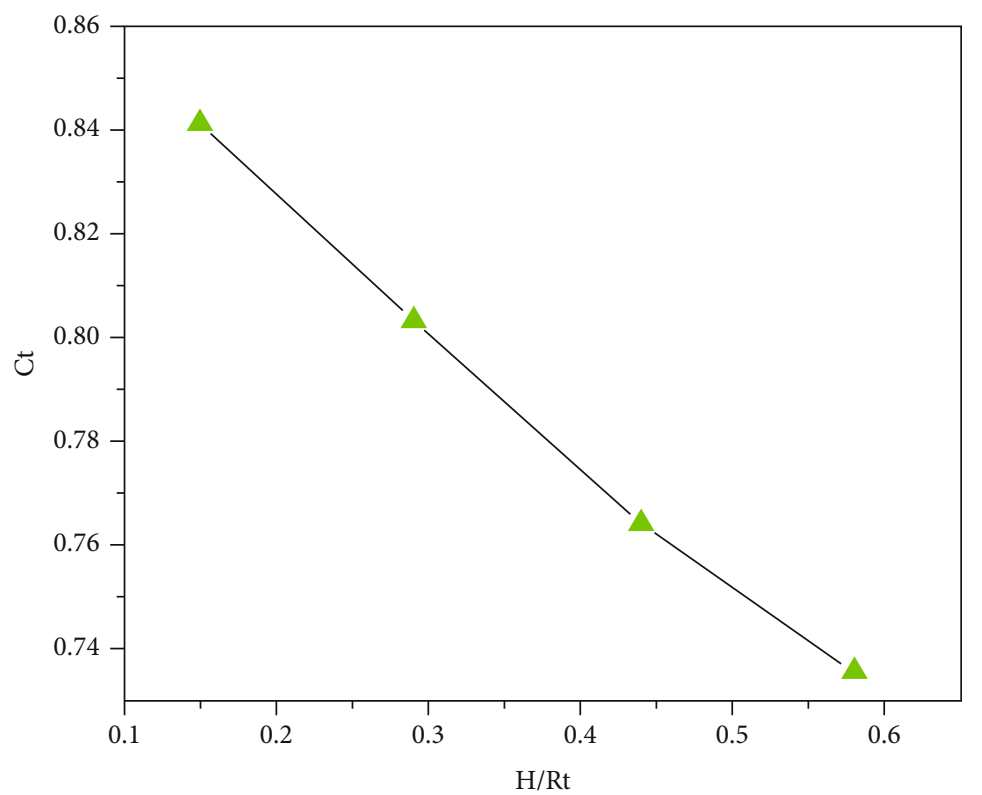

FIgURE 12: Thrust coefficients for diverse rod penetration heights $\left(\mathrm{NPR}=4.6\right.$ and $\left.X_{\mathrm{r}} / L_{\mathrm{d}}=0.8\right)$.

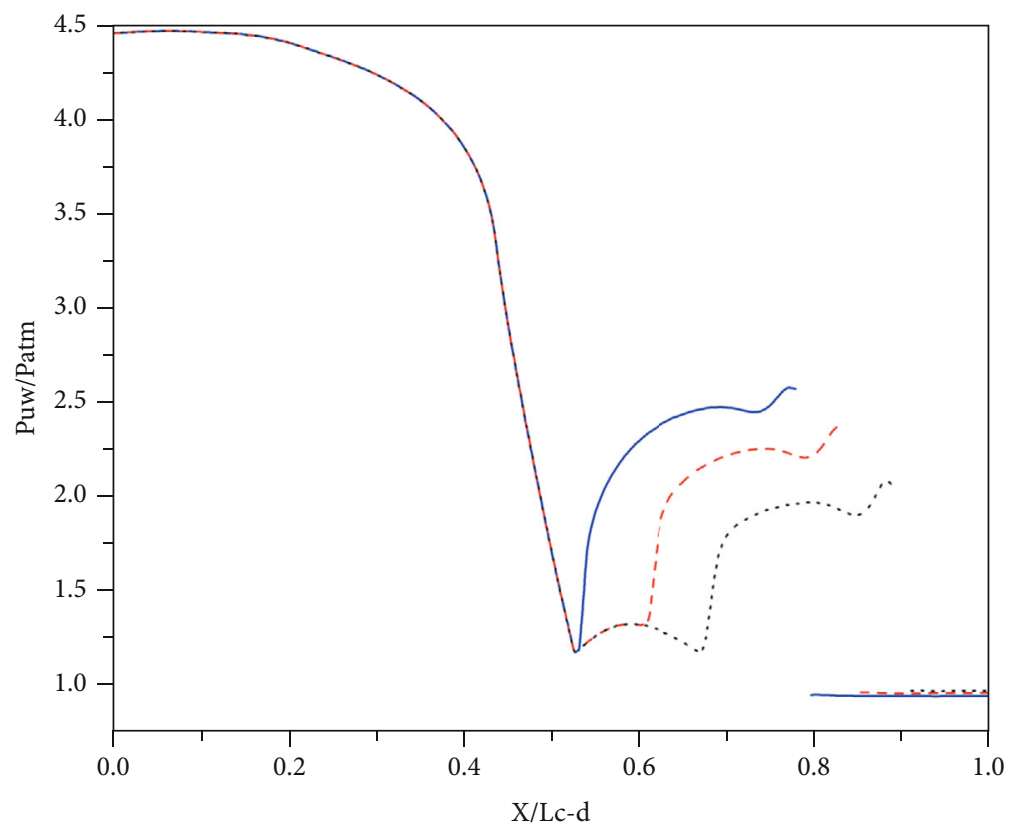

$\mathrm{Xr} / \mathrm{Ld}=0.6$

$-\ldots--. \mathrm{Xr} / \mathrm{Ld}=0.7$

…... Xr/Ld $=0.8$

FIGURE 13: Static pressure distributions along the top wall for diverse rod locations (NPR $=4.6$ and $H / R_{\mathrm{t}}=0.58$ ).

It demonstrates that the above phenomena match very well. Thus, the SST $k-\omega$ turbulence model is used for all CFD work.

2.5. Grid Sensitivity Study. A grid sensitivity study is necessary to acquire the fewest grid cells that can gain exact solutions. One coarse grid 1, one medium grid 2, and another fine grid 3 are tested, which have 0.18 million, 0.36 million, and 0.73 million cells. These three grids are imported in the Fluent, and the same settings are conducted as mentioned in Section 2.3. After obtaining reliable convergent solutions, three static pressure distributions along the top wall are compared to each other, as illustrated in Figure 7. The static pressure is normalized by the atmosphere pressure, $P_{\text {atm }}$ $\left(P_{\mathrm{atm}}=101325 \mathrm{~Pa}\right)$, and the axial distance, $X$, is normalized by the length of the diverging part of the supersonic nozzle, 


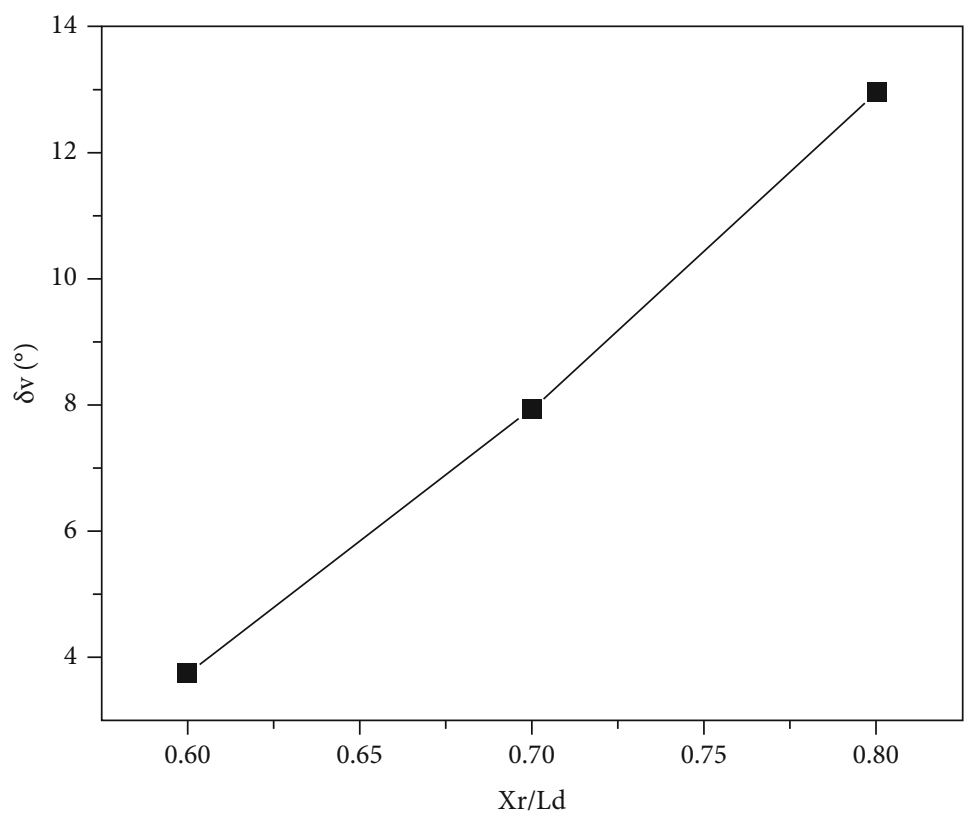

Figure 14: Vectoring angles for diverse rod locations $\left(\mathrm{NPR}=4.6\right.$ and $\mathrm{H} / \mathrm{R}_{\mathrm{t}}=0.58$ ).

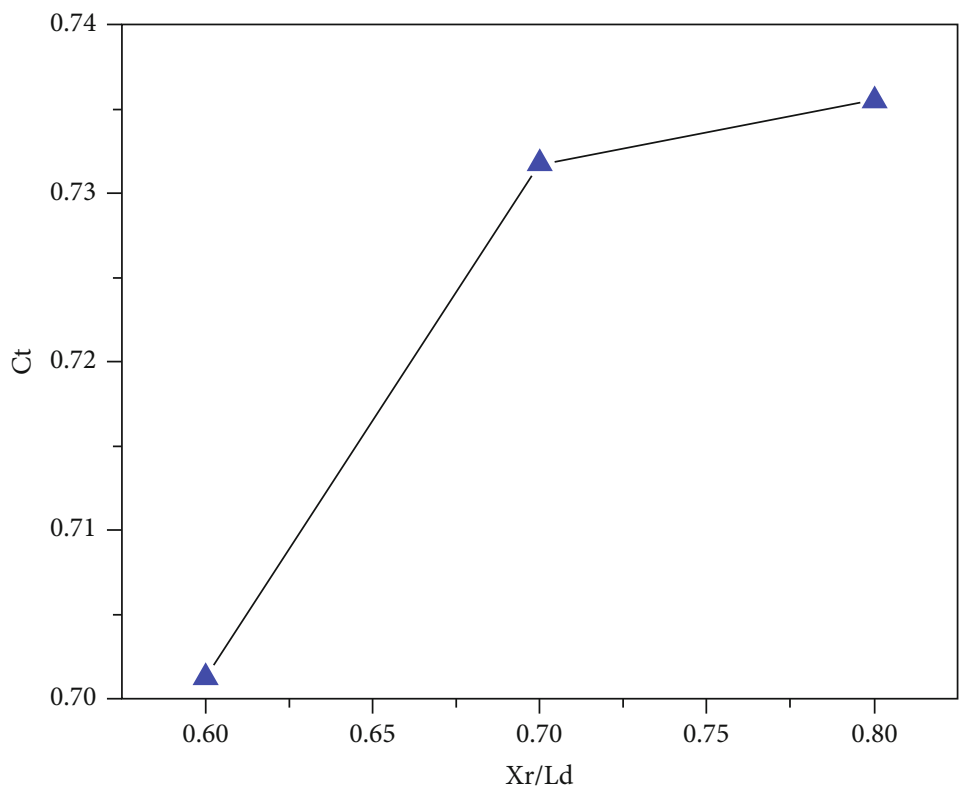

Figure 15: Thrust coefficients for diverse rod locations $\left(\mathrm{NPR}=4.6\right.$ and $\left.H / R_{\mathrm{t}}=0.58\right)$.

$L_{\mathrm{d}}$. It is seen that the normalized static pressure of grid 2 almost overlaps that of grid 3; however, the normalized static pressure of grid 1 has a certain gap in comparison to another two grids. From the above analysis of the grid sensitivity, the grid with 0.36 million cells is most suitable for the current CFD work.

\section{Results and Discussion}

The current work is aimed at elucidating detailed effects of the rod penetration height and rod location for the first time based upon advanced CFD means. Flow visualizations and quantitative analyses are elucidated in full detail. In Section 3.1 , the rod insertion goes deeper and deeper to explore control effectiveness. In Section 3.2, three diverse rod positions are investigated to illuminate the influence of the rod location on vectoring performance. In Section 3.3, unsteady flow oscillations are studied for different rod penetration heights to demonstrate the impacts of the separation bubbles ahead of the rod.

3.1. Steady-State Effect of Rod Penetration Height. Figure 8 shows static pressure distributions along with the top wall for diverse rod penetration heights at NPR $=4.6$ and $X_{\mathrm{r}} / L_{\mathrm{d}}$ 


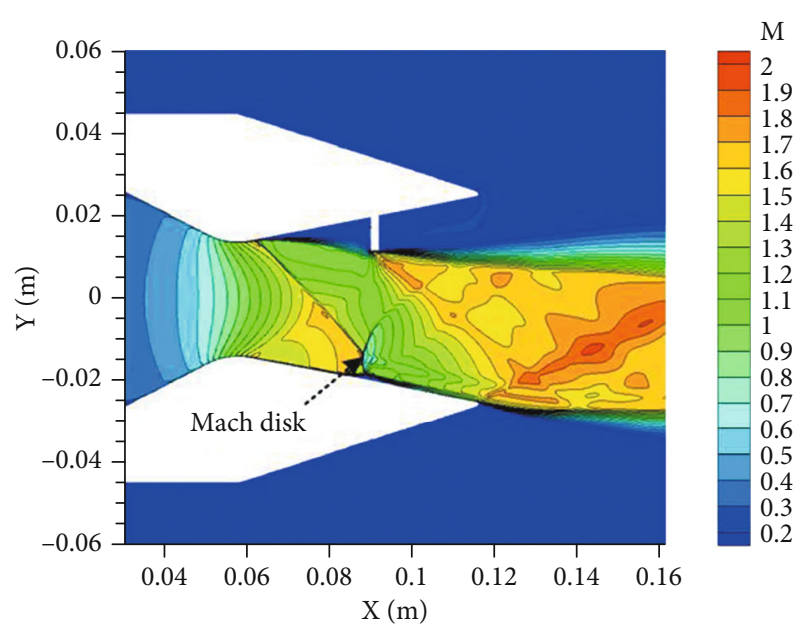

(a)

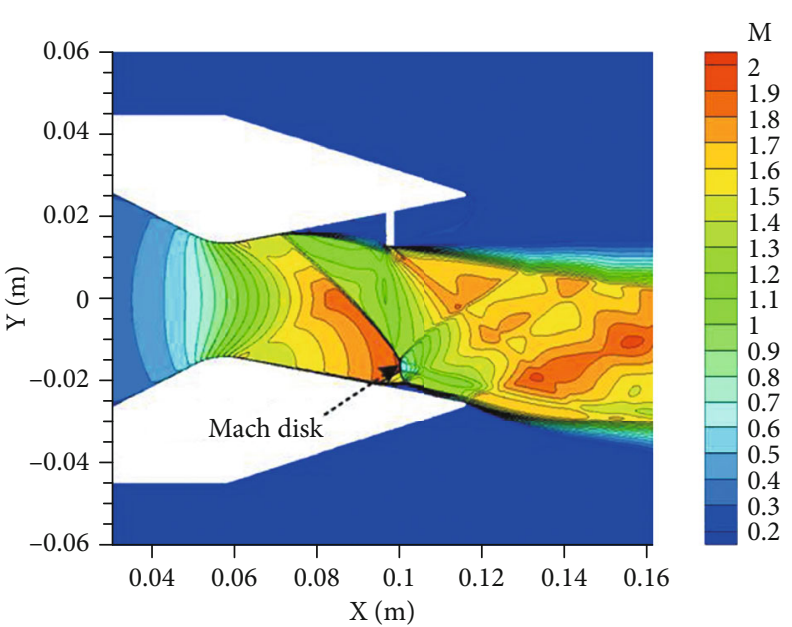

(b)

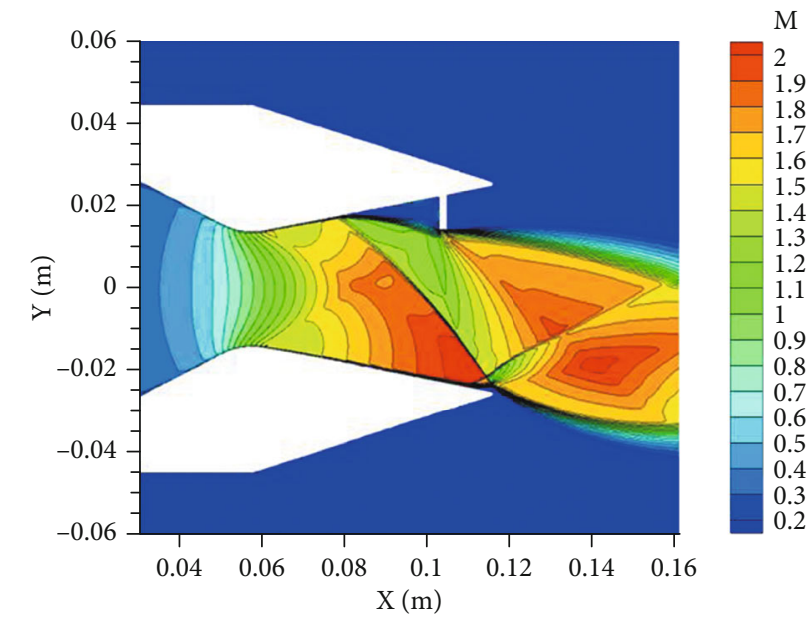

(c)

Figure 16: Mach contours for diverse rod locations $\left(\mathrm{NPR}=4.6\right.$ and $\left.H / R_{\mathrm{t}}=0.58\right)$ : (a) $X_{\mathrm{r}} / L_{\mathrm{d}}=0.6$; (b) $X_{\mathrm{r}} / L_{\mathrm{d}}=0.7$; (c) $X_{\mathrm{r}} / L_{\mathrm{d}}=0.8$.

$=0.8$. The horizontal axis represents normalized distance using the nozzle length, $L_{\mathrm{c}-\mathrm{d}}$. The vertical axis denotes normalized pressure utilizing the standard atmosphere pressure, $P_{\text {atm }}$. Mach contours for diverse rod penetration heights are depicted in Figure 9 to qualitatively explain the flow-field phenomena. Up to $X / L_{\mathrm{c}-\mathrm{d}}=0.67$, all static pressures follow an identical trend that they isentropically decrease in front of the boundary layer separation point. During this time, the flow accelerates with an increase in the section area of the nozzle. Beyond $X / L_{\mathrm{c}-\mathrm{d}}=0.67$, the location of boundary layer separation moves downstream in turn with the decline in the rod penetration height. For each circumstance, the static pressure rises from the boundary layer separation point owing to the separation shock. Then, it goes up to form a plateau-shaped pressure because of the existence of a primary vortex. It needs to be emphasized that the plateau pressure is wider and higher for a larger rod penetration height, because of the enlarged primary vortex area. Subsequently, each static pressure suddenly jumps to its peak pressure because of the appearance of detached bow shock ahead of the rod. What needs to be emphasized is that the value of peak pressure becomes higher with an increase in the rod penetration height, due to the stronger detached bow shock. A small drop after the pressure peak is associated with an interaction with the stationary rod. Behind the rod, the constant static pressure distribution is due to the formation of a downstream vortex, which is caused by the acceleration and deflection effects of expansion fans. Streaklines are exhibited in Figure 10 to visualize vortex variations ahead of the rod. It shows that the scale of the upstream vortex region prominently enlarges with an increase in rod penetration height, corresponding to the upstream movement of the boundary layer separation location.

Figure 11 shows vectoring angles for diverse rod penetration heights at $\mathrm{NPR}=4.6$ and $X_{\mathrm{r}} / L_{\mathrm{d}}=0.8$. The vectoring angle remarkably increases with an increasing rod penetration height in the effective control range of the technique. Figure 12 shows thrust coefficients for diverse rod penetration heights at NPR $=4.6$ and $X_{\mathrm{r}} / L_{\mathrm{d}}=0.8$. The thrust coefficient declines with an increase in rod penetration height. It is owing to stronger induced shocks generated by a deeper rod insertion. 


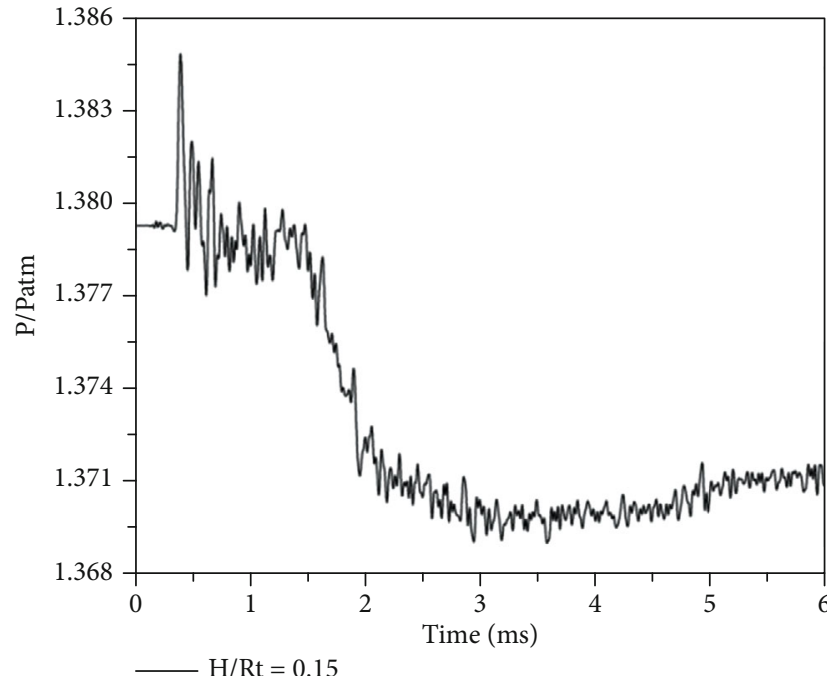

(a)

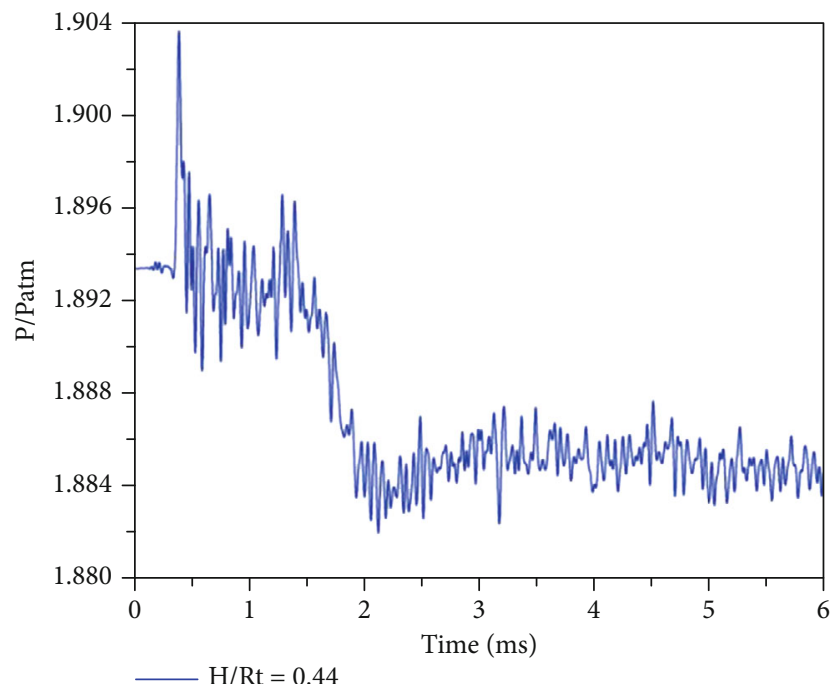

(c)

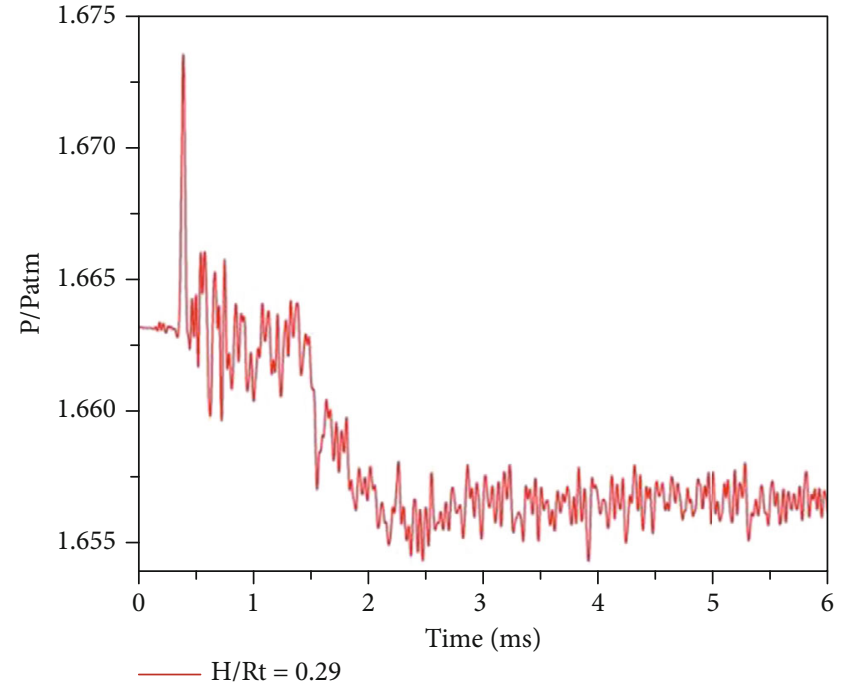

(b)

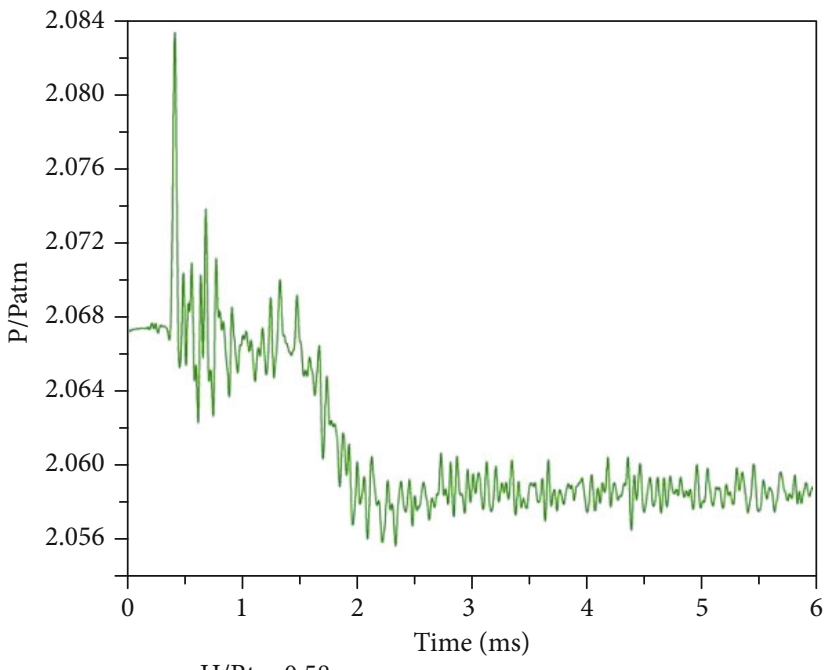

(d)

FIgURE 17: Pressure oscillations at the measurement point for different rod penetration heights $\left(\mathrm{NPR}=4.6\right.$ and $\left.X_{\mathrm{r}} / L_{\mathrm{d}}=0.8\right):(\mathrm{a}) H / R_{\mathrm{t}}=0.15$; (b) $H / R_{\mathrm{t}}=0.29 ;$ (c) $H / R_{\mathrm{t}}=0.44 ;$ (d) $H / R_{\mathrm{t}}=0.58$.

3.2. Steady-State Effect of Rod Location. The effects of rod location are investigated for an identical rod penetration height of $H / R_{\mathrm{t}}=0.58$ at $\mathrm{NPR}=4.6$. Figure 13 shows static pressure distributions along with the top wall for different rod locations. Until $X / L_{\mathrm{c}-\mathrm{d}}=0.53$, all static pressures follow the same tendency that they isentropically decrease in front of the boundary layer separation point. Beyond $X / L=0.53$, the location of boundary layer separation moves downstream with the downstream movement of the rod location towards the nozzle exit. Figure 14 shows the variation of vectoring angles for different rod locations at NPR $=4.6$ and $H / R_{\mathrm{t}}=0.58$. The vectoring angle continually increases with the downstream movement of the rod position towards the nozzle exit. It indicates that the rod position affects vectoring effectiveness appreciably. Moreover, a more downstream rod location can achieve a larger vectoring angle. Figure 15 shows thrust coefficients for diverse rod locations at NPR $=4.6$ and $H / R_{\mathrm{t}}=0.58$. The thrust coefficient increases continuously with a more downstream move of the rod location. As the rod location moves downstream, the Mach number of the nozzle flow is higher at the position of the shock generation. Generally, stronger shocks should be induced inside the nozzle flow field, resulting in more thrust loss; however, the transition from the Mach reflection to the regular reflection leads to the reduction of the thrust loss, as shown in Figure 16 [40]. Further observation of the Mach contour for different rod locations reveals that the length of the Mach disk for $X_{\mathrm{r}} / L_{\mathrm{d}}=0.6$ is larger than that of $X_{\mathrm{r}} / L_{\mathrm{d}}=0.7$, resulting in more thrust loss.

3.3. Unsteady-State Effect of Rod Penetration Height. Static pressure histories at the measurement point for different 


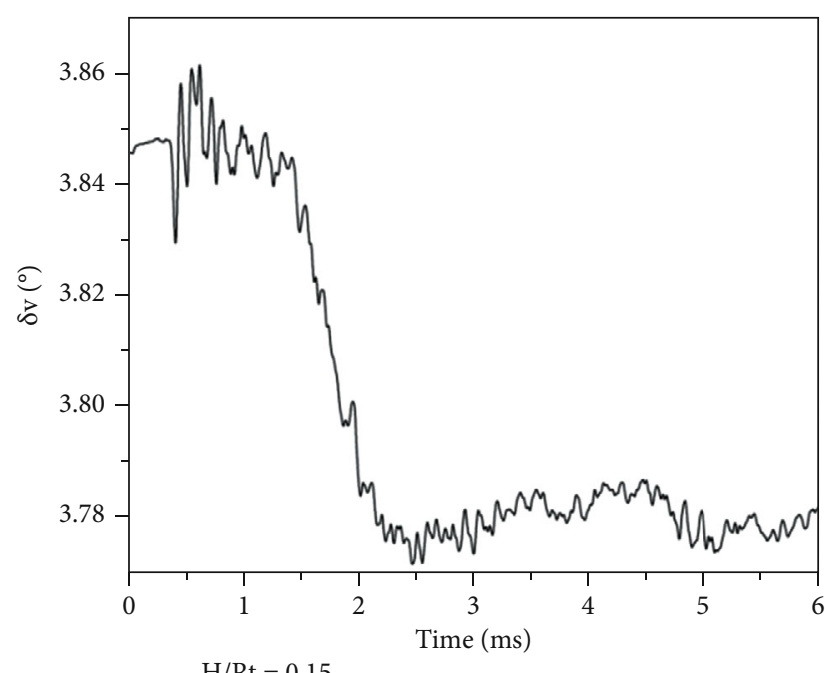

(a)

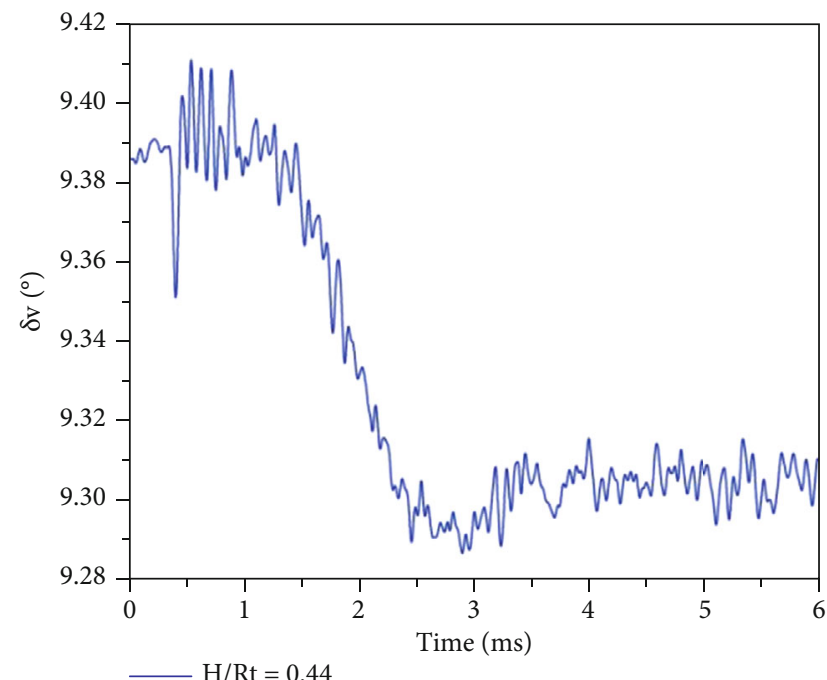

(c)

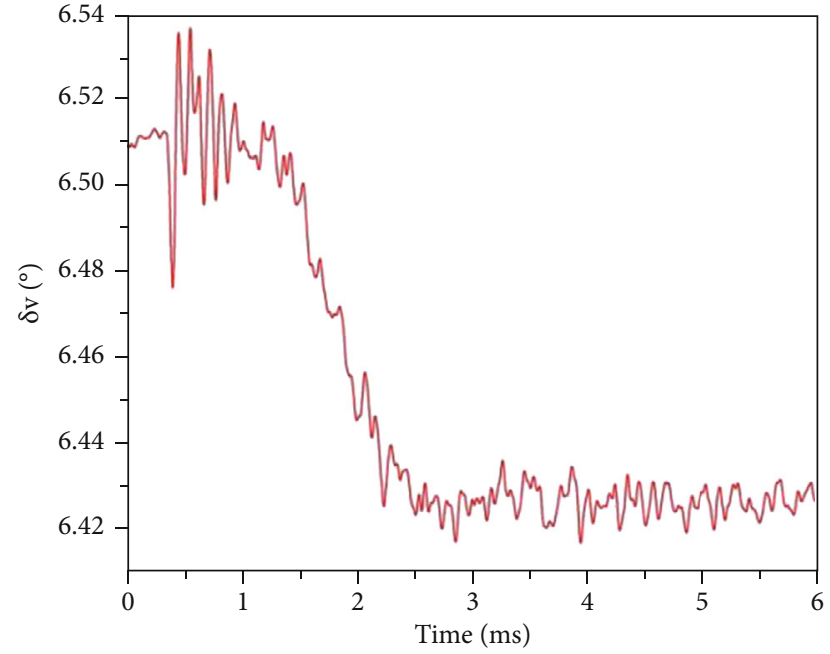

$\mathrm{H} / \mathrm{Rt}=0.29$

(b)

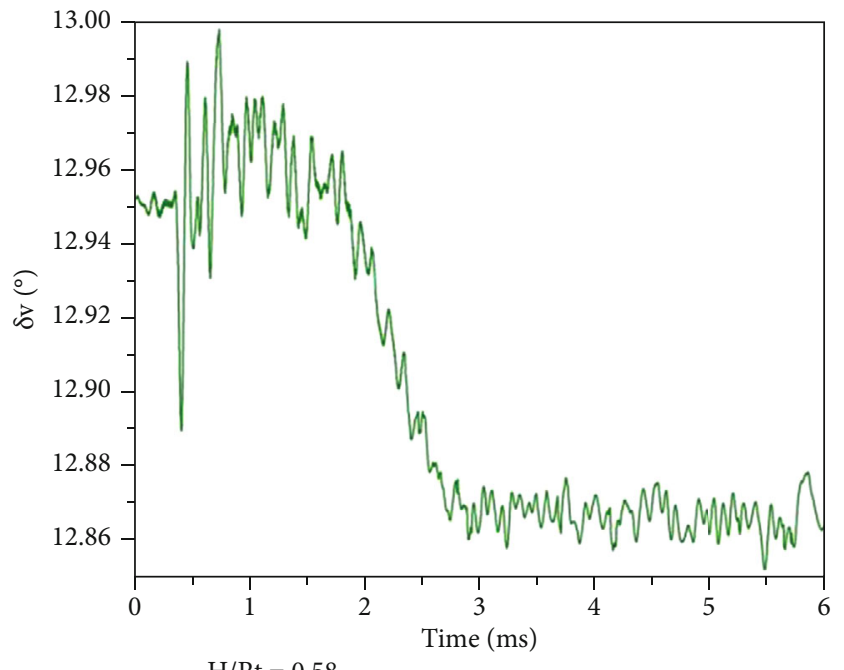

(d)

FIgURE 18: Vectoring angle oscillations for different rod penetration heights $\left(\mathrm{NPR}=4.6\right.$ and $\left.X_{\mathrm{r}} / L_{\mathrm{d}}=0.8\right)$ : (a) $H / R_{\mathrm{t}}=0.15$; (b) $H / R_{\mathrm{t}}=0.29$; (c) $H / R_{\mathrm{t}}=0.44 ;$ (d) $H / R_{\mathrm{t}}=0.58$.

rod penetration heights are shown in Figure 17. The penetrating rod results in the upstream anticlockwise separation bubbles. When the unsteady simulation time is less than $2 \mathrm{~ms}$, the pressure oscillation is more significant due to the initial transition from the steady simulation to the unsteady simulation. As the unsteady simulation time is beyond $2 \mathrm{~ms}$, the pressure oscillation becomes stable. Moreover, the static pressure level at the measurement point is higher for a larger rod penetration height. It reveals that flow oscillations always exist in real engineering applications. The unsteadystate effects caused by the expanding and shrinking of the vortices lead to flow oscillations. The calculated vectoring angle oscillations for different rod penetration heights are shown in Figure 18. The vectoring angle oscillation is mainly related to the flow oscillation caused by the upstream separation bubbles. It can be found that the vectoring angle oscillation occurs all the time, whereas the variation of the vectoring angle is very small. As a consequence, it proves that this rod thrust vector control method offers stable and effective control effectiveness under the circumstance of flow oscillations.

\section{Conclusions}

The vectoring control effectiveness using a simple rod for a 2-D supersonic nozzle is investigated for the first time based upon advanced CFD means. Published experimental results are used to validate the current CFD result. An excellent consistency between the CFD and the experimental results is obtained. Steady-state characteristics by changing the rod penetration height and rod location are qualitatively and quantitatively analyzed. Unsteady-state features are specifically studied for different rod penetration heights to illuminate the flow oscillations related to upstream 
separation bubbles. The conclusions reveal that the above factors affect vectoring performance appreciably. Clear schlieren images of the Mach contour and streaklines are obtained to illustrate the flow-field structures inside the supersonic nozzle. Significant conclusions are drawn as follows.

The applicability of the thrust vectoring approach is verified in overexpansion flow conditions. The rod penetration height influences system vectoring performance significantly. With an increasing rod penetration height, the boundary layer separation location moves upstream along the top nozzle wall. The vectoring angle increases, and the thrust coefficient decreases with an increase in rod penetration height. Using different rod penetration heights to control the variation angle is an effective and sensitive technology.

The rod position remarkably affects the direction of the jet deflection. Moreover, the vectoring angle and the thrust coefficient continually increase with the downstream movement of the rod location towards the nozzle exit.

Concerning the unsteady-state influence of diverse rod penetration heights, certain flow oscillations caused by the upstream separation bubbles are illustrated. However, these flow oscillations do not affect the vectoring angle oscillations remarkably. Therefore, the simple rod thrust vector control technology is proved as a stable and effective flow control method.

\section{Nomenclature}

$A_{\mathrm{e}}: \quad$ Nozzle exit area $\left(\mathrm{mm}^{2}\right)$

$C_{\mathrm{t}}$ : Thrust coefficient

$F_{\mathrm{i}}$ : Theoretically isentropic thrust $(\mathrm{N})$

$F_{\mathrm{r}}$ : $\quad$ Real thrust $(\mathrm{N})$

$F_{x}$ : Horizontal force $(\mathrm{N})$

$F_{y}: \quad$ Vertical force $(\mathrm{N})$

$H$ : $\quad$ Rod penetration height $(\mathrm{mm})$

$H_{\mathrm{e}}$ : Nozzle exit height $(\mathrm{mm})$

$H_{\mathrm{t}}$ : Nozzle throat height $(\mathrm{mm})$

L: $\quad$ Rod length ( $\mathrm{mm})$

$L_{\mathrm{c}-\mathrm{d}}$ : Converging-diverging length of the supersonic nozzle (mm)

$L_{\mathrm{d}}$ : Diverging part length of the supersonic nozzle $(\mathrm{mm})$

$L_{\mathrm{r}}$ : Distance between the original point to rod location (mm)

M: $\quad$ Mach number

$P: \quad$ Static pressure $(\mathrm{Pa})$

$P_{0}: \quad$ Total pressure $(\mathrm{Pa})$

$P_{\text {atm }}:$ Atmosphere pressure $(\mathrm{Pa})$

$P_{\mathrm{e}}$ : $\quad$ Area-weighted average pressure at the nozzle exit $(\mathrm{Pa})$

$P_{\mathrm{uw}}$ : Static pressure along the top nozzle wall $(\mathrm{Pa})$

$R_{\mathrm{g}}$ : $\quad$ Specific gas constant $(\mathrm{kJ} / \mathrm{kg} \mathrm{K})$

$R_{\mathrm{t}}$ : Nozzle throat radius $(\mathrm{mm})$

$T: \quad$ Static temperature $(\mathrm{K})$

$T_{0}$ : Total temperature $(\mathrm{K})$

$U_{\mathrm{ex}}: \quad x$-component velocity at the nozzle exit $(\mathrm{m} / \mathrm{s})$

$U_{\text {ey }}: \quad y$-component velocity at the nozzle exit $(\mathrm{m} / \mathrm{s})$

$\gamma: \quad$ Specific heat ratio (1.4 for air)

$\delta_{\mathrm{v}}: \quad$ Vectoring angle $\left({ }^{\circ}\right)$

$\dot{m}$ : Mass flow rate $(\mathrm{kg} / \mathrm{s})$ $\rho: \quad$ Density $\left(\mathrm{kg} / \mathrm{m}^{3}\right)$.

Abbreviations

2-D: Two-dimensional

AUSM: Advection upstream splitting method

CFD: Computational fluid dynamics

NPR: $\quad$ Nozzle pressure ratio $\left(\mathrm{NPR}=P_{0} / P_{\mathrm{atm}}\right)$

RANS: Reynolds-averaged Navier-Stokes

S-A: Spalart-Allmaras

SST: $\quad$ Shear-stress transport.

\section{Conflicts of Interest}

The authors declare that they have no conflicts of interest.

\section{Acknowledgments}

The work is supported by the National Research Foundation of Korea (NRF).

\section{References}

[1] K. X. Wu and H. D. Kim, "A fluidic thrust vector control using the bypass flow in a dual throat nozzle," Journal of Mechanical Science and Technology, vol. 35, no. 8, pp. 3435-3443, 2021.

[2] H. J. Hollstein, "Jet tab thrust vector control," Journal of Spacecraft and Rockets, vol. 2, no. 6, pp. 927-930, 1965.

[3] A. Banazadeh, F. Saghafi, M. Ghoreyshi, and P. Pilidis, "Experimental and computational investigation into the use of co-flow fluidic thrust vectoring on a small gas turbine," Aeronautical Journal, vol. 112, no. 1127, pp. 17-25, 2008.

[4] H. G. Sung and J. Y. Heo, "Fluidic thrust-vector control of supersonic jet using coflow injection," Journal of Propulsion and Power, vol. 28, no. 4, pp. 858-861, 2012.

[5] K. X. Wu, H. D. Kim, and Y. Z. Jin, "Fluidic thrust vector control based on counter-flow concept," Proceedings of the Institution of Mechanical Engineers, Part G: Journal of Aerospace Engineering, vol. 233, pp. 1412-1422, 2019.

[6] K. X. Wu, Y. Jin, and H. D. Kim, "Hysteretic behaviors in counter-flow thrust vector control," ASCE Journal of Aerospace Engineering, vol. 32, no. 4, article 04019041, 2019.

[7] K. X. Wu, G. Zhang, T. H. Kim, and H. D. Kim, "Numerical parametric study on three-dimensional rectangular counterflow thrust vectoring control," Proceedings of the Institution of Mechanical Engineers, Part G: Journal of Aerospace Engineering, vol. 234, no. 16, pp. 2221-2247, 2020.

[8] K. X. Wu, T. H. Kim, and H. D. Kim, "Sensitivity analysis of counterflow thrust vector control with a three-dimensional rectangular nozzle," Journal of Aerospace Engineering, vol. 34, no. 1, article 04020107, 2021.

[9] K. X. Wu and H. Dong Kim, "Numerical study on the shock vector control in a rectangular supersonic nozzle," Proceedings of the Institution of Mechanical Engineers, Part G: Journal of Aerospace Engineering, vol. 233, no. 13, pp. 4943-4965, 2019.

[10] D. F. Yan, Z. J. Wei, K. Xie, and N. F. Wang, "Simulation of thrust control by fluidic injection and pintle in a solid rocket motor," Aerospace Science and Technology, vol. 99, article 105711, 2020.

[11] K. X. Wu, H. D. Kim, and H. D. Kim, “Theoretical and numerical analyses of aerodynamic characteristics on shock vector 
control," Journal of Aerospace Engineering, vol. 33, no. 5, article 04020050, 2020.

[12] C. C. Guo, Z. J. Wei, K. Xie, and N. F. Wang, "Thrust control by fluidic injection in solid rocket motors," Journal of Propulsion and Power, vol. 33, no. 4, pp. 815-829, 2017.

[13] R. Y. Deng and H. D. Kim, "A study on the thrust vector control using a bypass flow passage," Proceedings of the Institution of Mechanical Engineers, Part G: Journal of Aerospace Engineering, vol. 229, no. 9, pp. 1722-1729, 2015.

[14] R. Y. Deng, T. Setoguchi, and H. D. Kim, "Large eddy simulation of shock vector control using bypass flow passage," International Journal of Heat and Fluid Flow, vol. 62, pp. 474-481, 2016.

[15] K. A. Deere, "Summary of fluidic thrust vectoring research conducted at NASA Langley Research Center," in 21st AIAA Applied Aerodynamics Conference, pp. 1-18, Orlando, FL, 2003.

[16] K. A. Deere, B. L. Berrier, J. D. Flamm, and S. K. Johnson, "Computational study of fluidic thrust vectoring using separation control in a nozzle," in 21st AIAA applied aerodynamics conference, pp. 1-11, Orlando, FL, 2003.

[17] P. J. Yagle, D. N. Miller, K. B. Ginn, and J. W. Hamstra, "Demonstration of fluidic throat skewing for thrust vectoring in structurally fixed nozzles," Journal of Engineering for Gas Turbines and Power, vol. 123, no. 3, pp. 502-507, 2001.

[18] M. Ferlauto and R. Marsilio, "Numerical simulation of fluidic thrust-vectoring," Aerotecnica Missili \& Spazio, vol. 95, no. 3, pp. 153-162, 2016.

[19] K. X. Wu and H. D. Kim, "Study on fluidic thrust vector control based on dual-throat concept," Journal of the Korean Society of Propulsion Engineers, vol. 23, no. 1, pp. 24-32, 2019.

[20] R. Gu, J. Xu, and S. Guo, "Experimental and numerical investigations of a bypass dual throat nozzle," Journal of Engineering for Gas Turbines and Power, vol. 136, no. 8, pp. 1-6, 2014.

[21] Y. S. Wang, J. L. Xu, S. Huang, Y. C. Lin, and J. J. Jiang, "Computational study of axisymmetric divergent bypass dual throat nozzle," Aerospace Science and Technology, vol. 86, pp. 177190, 2019.

[22] H. G. Sung and Y. S. Hwang, "Thrust-vector characteristics of jet vanes arranged in $\mathrm{X}$-formation within a shroud," Journal of Propulsion and Power, vol. 20, no. 3, pp. 501-508, 2004.

[23] S. R. Wassom, L. C. Faupell, and T. Perley, "Integrated aerofin/thrust vector control for tactical missiles," Journal of Propulsion and Power, vol. 7, no. 3, pp. 374-381, 1991.

[24] F. S. Kong, Y. Z. Jin, and H. D. Kim, "Thrust vector control of supersonic nozzle flow using a moving plate," Journal of Mechanical Science and Technology, vol. 30, no. 3, pp. 12091216, 2016.

[25] R. F. Cong, Y. D. Ye, Z. L. Zhao, J. Q. Wu, and C. F. Zhang, "Numerical research on jet tab thrust vector nozzle aerodynamic characteristics," Journal de Physique, vol. 1300, pp. 16, 2019.

[26] R. Cavalleri, W. Tiarn, and H. Readey, "Thrust vector control using movable probes," in 28th Aerospace sciences meeting, pp. 90-0562, Reno,NV,U.S.A., 1990.

[27] W. Tiarn and R. Cavalleri, "CFD evaluation of an advanced thrust vector control concept," in AIAA 26th Joint propulsion conference, pp. 90-1900, Orlando,FL,U.S.A., July 1990.
[28] R. Cavalleri and W. Tiarn, "Experimental and theoretical comparison of the probe thrust vector control concept," in AIAA 27th Joint propulsion conference, pp. 91-2476-CP, Sacramento, CA, June 1991.

[29] R. J. Cavalleri, "Fluid shielded movable strut for missile and rocket thrust vector control," 1992, Patent Number: 5125596.

[30] D. Mokhtari, M. Hojaji, and M. Afrand, "Experimental investigation of the effect of cylindrical protuberance with different penetration the thrust vector a C-D nozzle in supersonic regime," Modares Mechanical Engineering., vol. 19, pp. 1145$1154,2019$.

[31] M. R. Babaeyan, "Experimental investigation of the penetration effects of opposite dual protuberances on thrust vector of a supersonic C-D nozzle," Modares Mechanical Engineering, vol. 19, pp. 1741-1750, 2019.

[32] A. L. Srinivas and B. T. V. Sridhar, "Experimental study of the wall pressure distribution in a convergent-divergent nozzle with strut injection," Fluid Dynamics, vol. 55, no. 2, pp. 279290, 2020.

[33] A. Hadjadj and J. P. Dussauge, "Shock wave boundary layer interaction," Shock Waves, vol. 19, no. 6, pp. 449-452, 2009.

[34] D. F. Yan, Z. J. Wei, K. Xie, C. C. Guo, W. Tang, and N. F. Wang, "Study of the vortex structure of a subsonic jet in an axisymmetric transonic nozzle," Physics of Fluids, vol. 32, no. 7 , article $076109,2020$.

[35] J. C. Westkaemper, "Turbulent boundary-layer separation ahead of cylinders," AIAA Journal, vol. 6, no. 7, pp. 1352 1355, 1968.

[36] D. S. Dolling and S. M. Bogdonoff, "Scaling of interactions of cylinders with supersonic turbulent boundary layers," AIAA Journal, vol. 19, no. 5, pp. 655-657, 1981.

[37] O. Özcan and B. K. Yüceil, "Cylinder-induced shock-wave boundary layer interaction,” AIAA Journal, vol. 30, no. 4, pp. 1130-1132, 1992.

[38] K. A. Waithe and K. A. Deere, "Experimental and computational investigation of multiple injection ports in a convergent-divergent nozzle for fluidic thrust vectoring," in 21th AIAA applied aerodynamics conference, pp. 1-14, Orlando, FL, USA, June 2003.

[39] K. X. Wu, S. K. Raman, V. R. P. Sethuraman, G. Zhang, and H. D. Kim, "Effect of the wall temperature on Mach stem transformation in pseudo-steady shock wave reflections," International Journal of Heat and Mass Transfer, vol. 147, p. 118927, 2020.

[40] K. X. Wu, G. Zhang, and H. D. Kim, "Study on the Mach and regular reflections of shock wave," Journal of Visualization, vol. 22, no. 2, pp. 283-303, 2019. 